\title{
Uniformly boundedness of a class of non-linear differential equations of third order with multiple deviating arguments
}

\author{
Cemil Tunç, Hilmi Ergören \\ Department of Mathematics, Faculty of Sciences, \\ Yüzüncü Yıl University, \\ 65080, Van, TURKEY \\ email: cemtunc@yahoo.com, hergoren@yahoo.com
}

\begin{abstract}
This paper deals with a certain third-order non-linear differential equation with multiple deviating arguments. Some sufficient conditions are set up for all solutions and their derivatives to be uniformly bounded.
\end{abstract}

\section{RESUMEN}

En este artículo se estudia un tipo de ecuaciones diferenciales no lineales de tercer orden con argumentos de desviación múltiple. Se establecen algunas condiciones suficientes para que todas las soluciones y sus derivadas sean uniformemente acotadas.

Keywords and Phrases: Non-linear differential equation; third order; multiple deviating arguments; bounded solutions.

2010 AMS Mathematics Subject Classification: 34C25; 34K13; 34K25. 


\section{Introduction}

Consider the following third order nonlinear differential equation with multiple deviating arguments

$$
x^{\prime \prime \prime}(t)+f_{1}(t, x(t)) x^{\prime \prime}(t)+f_{2}(t, x(t)) x^{\prime}(t)+g_{0}(t, x(t))+\sum_{i=1}^{n} g_{i}\left(t, x\left(t-\tau_{i}(t)\right)=p(t),\right.
$$

where $f_{1}, f_{2}$ and $g_{i}(i=0,1,2, \ldots, n)$ are continuous functions on $R^{+} \times R, \tau_{i}(t) \geq 0(i=1,2, \ldots, n)$ and $p(t)$ are bounded continuous functions on $R$ and $R^{+}=[0,+\infty)$, respectively.

Define $y(t)=\frac{d x(t)}{d t}+\alpha_{1} x(t)$ and $z(t)=\frac{d y(t)}{d t}+\alpha_{2} y(t)$, where $\alpha_{1}$ and $\alpha_{2}$ are some constants. Then, we can transform (1.1) into the following system

$$
\begin{aligned}
\frac{d x(t)}{d t}= & -\alpha_{1} x(t)+y(t) \\
\frac{d y(t)}{d t}= & -\alpha_{2} y(t)+z(t), \\
\frac{d z(t)}{d t}= & -\left(f_{1}(t, x(t))-\alpha_{1}-\alpha_{2}\right) z(t) \\
& +\left(\left(f_{1}(t, x(t))-\alpha_{1}\right)\left(\alpha_{1}+\alpha_{2}\right)-f_{2}(t, x(t))-\alpha_{2}^{2}\right) y(t) \\
& +\left(\left(\alpha_{1}-f_{1}(t, x(t))\right) \alpha_{1}^{2}+f_{2}(t, x(t)) \alpha_{1}\right) x(t)-g_{0}(t, x(t)) \\
& -\sum_{i=1}^{n} g_{i}\left(t, x\left(t-\tau_{i}(t)\right)+p(t) .\right.
\end{aligned}
$$

In applied science some practical problems are associated with higher-order nonlinear differential equations, such as nonlinear oscillations (Afuwape et al. ${ }^{[1]}$, Andres ${ }^{[2]}$ and Fridedrichs ${ }^{[3]}$ ), electronic theory $\left(\operatorname{Rauch}^{[4]}\right)$, biological models and other models (Cronin ${ }^{[5]}$ and Gopalsamy ${ }^{[6]}$ ). Just as above, in the past few decades, the study for third order differential equations has been paid attention by many scholars. Many results relative to the stability, boundedness of solutions of third order differential equations with delays or without delays have been obtained (see $\mathrm{Li}^{[7]}$, Murakami $^{[8]}$, Ademola et al. ${ }^{[9]}$, Tunç and Ergören ${ }^{[10]}$, Tunç ${ }^{[11-13]}$ and references therein). However, to the best of our knowledge, no authors have considered the boundedness of solutions of third order differential equations with multiple deviating arguments in non-Liapunov sense, in spite of the fact that some authors (see Afuwape and Castellanos ${ }^{[14]}$, Gao and $\mathrm{Liu}^{[15]}$, and $\mathrm{Yu}$ and Zhao ${ }^{[16]}$ ) have obtained some results for the third order ones with a deviating argument and second order ones with multiple deviating arguments. Thus, it is worthwhile to continue to the investigation of the boundedness of solutions of (1.1) in this case.

The main objective of this paper is to study the uniformly boundedness of solutions of (1.1). We will establish some sufficient conditions satisfying the solutions of (1.1) to be uniformly bounded. Our result is new and complement to previously known results. In particular, an example is also given to illustrate the effectiveness of the new result. 


\section{Definitions and Assumptions}

We assume that $h=\max _{1 \leq i \leq n}\left\{\sup _{t \in R} \tau_{i}(t)\right\} \geq 0$. Let $C([-h, 0], R)$ denote the space of continuous functions $\phi:[-\mathrm{h}, 0] \rightarrow \mathrm{R}$ with the supremum norm $\|$.$\| . It is known in Burton { }^{[17]}$, Hale ${ }^{[18]}$ and Kuang ${ }^{[19]}$ that there exists a solution of (1.2) on an interval $[0, T)$ satisfying the initial condition and (1.1) on $[0, T)$ for $g_{i}(i=0,1,2, \ldots, n), \phi, f_{1}, f_{2}, p$ and $\tau_{i}(t)(i=1,2, \ldots, n)$ continuous, given a continuous initial function $\phi \in C([-h, 0], R)$ and a vector $\left(y_{0}, z_{0}\right) \in R^{2}$. If the solution remains bounded, then $T=+\infty$. We denote such a solution by $(x(t), y(t), z(t))=$ $\left(x\left(t, \phi, y_{0}, z_{0}\right), y\left(t, \phi, y_{0}, z_{0}\right), z\left(t, \phi, y_{0}, z_{0}\right)\right)$, where $y(s)=y(0)$ and $z(s)=z(0)$ for all $s \in[-h, 0]$. Then, it follows that $(x(t), y(t), z(t))$ can be defined on $[-h,+\infty)$.

Definition. Solutions of (1.2) are called uniformly bounded (UB) if for each $\mathrm{B}_{1}>0$ there is a $B_{2}>0$ such that $\left(\phi, y_{0}, z_{0}\right) \in C([-h, 0], R) \times R^{2}$ and $\|\phi\|+\left\|y_{0}\right\|+\left\|z_{0}\right\| \leq B_{1}$ imply that $\left|x\left(t, \phi, y_{0}, z_{0}\right)\right|+\left|y\left(t, \phi, y_{0}, z_{0}\right)\right|+\left|z\left(t, \phi, y_{0}, z_{0}\right)\right| \leq B_{2}$ for all $t \in R^{+}$.

In this work, we also assume that the following conditions hold:

i) $\left|\left(\left(\alpha_{1}-f_{1}(t, u)\right) \alpha_{1}^{2}+f_{2}(t, u) \alpha_{1}\right) u-g_{0}(t, u)\right| \leq L_{0}|u|+q_{0}$ for all $u \in R$ and $t \geq 0$,

ii) $\left|g_{1}(t, u)\right| \leq L_{1}|u|+q_{1},\left|g_{2}(t, u)\right| \leq L_{2}|u|+q_{2}, \ldots,\left|g_{n}(t, u)\right| \leq L_{n}|u|+q_{n}$ for all $u \in R$ and $t \geq 0$,

iii) $\alpha_{3}=\inf _{t \geq 0}\left(f_{1}(t, u)-\alpha_{1}-\alpha_{2}\right)$

$$
-\sup _{t \geq 0}\left|\left(f_{1}(t, u)-\alpha_{1}\right)\left(\alpha_{1}+\alpha_{2}\right)-f_{2}(t, u)-\alpha_{2}^{2}\right|>\sum_{i=0}^{n} L_{i}, \text { where } \alpha_{1}>1, \alpha_{2}>1, \alpha_{3}>0
$$

are some constants, and $L_{i}$ and $q_{i}(i=0,1,2, \ldots, n)$ are nonnegative constants.

\section{$3 \quad$ Main Result}

Theorem 1. Suppose (i)-(iii) hold. Then, solutions of (1.2) are uniformly bounded.

Proof. Let $(x(t), y(t), z(t))=\left(x\left(t, \phi, y_{0}, z_{0}\right), y\left(t, \phi, y_{0}, z_{0}\right), z\left(t, \phi, y_{0}, z_{0}\right)\right)$ be a solution of system (1.2) defined on $[0, T)$. We may assume that $T=+\infty$ since the following estimates give a priori bound on $(x(t), y(t), z(t))$.

Calculating the upper right derivative of $|x(s)|,|y(s)|$ and $|z(s)|$, in view of (i) - (iii), we have

$$
\begin{aligned}
\mathrm{D}^{+}(|x(\mathrm{~s})|)_{s=\mathrm{t}} & =\operatorname{sgn}(x(\mathrm{t}))\left\{-\alpha_{1} x(\mathrm{t})+\mathrm{y}(\mathrm{t})\right\} \\
& \leq-\alpha_{1}|x(\mathrm{t})|+|\mathrm{y}(\mathrm{t})| \\
\mathrm{D}^{+}(|\mathrm{y}(\mathrm{s})|)_{s=\mathrm{t}} & =\operatorname{sgn}(\mathrm{y}(\mathrm{t}))\left\{-\alpha_{2} \mathrm{y}(\mathrm{t})+z(\mathrm{t})\right\} \\
& \leq-\alpha_{2}|\mathrm{y}(\mathrm{t})|+|z(\mathrm{t})|
\end{aligned}
$$


and

$$
\begin{aligned}
D^{+}(|z(s)|)_{s=t}= & \operatorname{sgn}(z(t))\left\{-\left(f_{1}(t, x(t))-\alpha_{1}-\alpha_{2}\right) z(t)\right. \\
& +\left(\left(f_{1}(t, x(t))-\alpha_{1}\right)\left(\alpha_{1}+\alpha_{2}\right)-f_{2}(t, x(t))-\alpha_{2}^{2}\right) y(t) \\
& +\left(\left(\alpha_{1}-f_{1}(t, x(t))\right) \alpha_{1}^{2}+f_{2}(t, x(t)) \alpha_{1}\right) x(t)-g_{0}(t, x(t)) \\
& -\sum_{i=1}^{n} g_{i}\left(t, x\left(t-\tau_{i}(t)\right)+p(t)\right\} \\
\leq \quad & -i_{t \geq 0}\left(f_{1}(t, x(t))-\alpha_{1}-\alpha_{2}\right)|z(t)| \\
& +\sup _{t \geq 0}\left|\left(f_{1}(t, x(t))-\alpha_{1}\right)\left(\alpha_{1}+\alpha_{2}\right)-f_{2}(t, x(t))-\alpha_{2}^{2}\right||y(t)| \\
& +L_{0}|x(t)|+\sum_{i=1}^{n} L_{i}\left|x\left(t-\tau_{i}(t)\right)\right|+\sum_{i=0}^{n} q_{i}+|p(t)| .
\end{aligned}
$$

Let

$$
M(t)=\max _{-h \leq s \leq t}\{\max \{|x(s)|,|y(s)|,|z(s)|\}\},
$$

where $y(s)=y(0), z(s)=z(0)$ for all $-h \leq s \leq 0$. It is clear that $\max \{|x(t)|,|y(t)|,|z(t)|\} \leq M(t)$ and $M(t)$ is non-decreasing for $t \geq-h$. Now, we consider the following two cases:

Case I):

$$
M(t)>\max \{|x(t)|,|y(t)|,|z(t)|\}
$$

for all $t \geq 0$, then we claim that

$$
M(t) \equiv M(0)
$$

is a constant for all $t \geq 0$.

By contrapositive, assume (3.6) does not hold, then, there exists $t_{1}>0$ such that $M\left(t_{1}\right)>$ $M(0)$.

Here $\max \{|x(t)|,|y(t)|,|z(t)|\} \leq M(0)$ for all $-h \leq t \leq 0$ and there exists $\beta \in\left(0, t_{1}\right)$ such that $\max \{|x(\beta)|,|y(\beta)|,|z(\beta)|\}=M\left(t_{1}\right) \geq M(\beta)$ which contradicts (3.5). This contradiction implies that (3.6) holds. It follows that there exists $t_{2}>0$ such that $\max \{|x(t)|,|y(t)|,|z(t)|\} \leq M(t)=$ $M(0)$ for all $t \geq t_{2}$.

Case II): There is a point $t_{0} \geq 0$ such that $M\left(t_{0}\right)=\max \left\{\left|x\left(t_{0}\right)\right|,\left|y\left(t_{0}\right)\right|,\left|z\left(t_{0}\right)\right|\right\}$. Let $\eta=$ $\min \left\{\alpha_{1}-1, \alpha_{2}-1, \alpha_{3}-\sum_{i=0}^{n} L_{i}\right\}>0$ and $\theta=\sum_{i=0}^{n} q_{i}+\sup _{t \in R^{+}}|p(t)|+1$ be constants, where $t \geq 0$. Then, if $M\left(t_{0}\right)=\max \left\{\left|x\left(t_{0}\right)\right|,\left|y\left(t_{0}\right)\right|,\left|z\left(t_{0}\right)\right|\right\}=\left|x\left(t_{0}\right)\right|$, then we obtain

$$
\begin{aligned}
\mathrm{D}^{+}(|x(s)|)_{s=t_{0}} & \leq-\alpha_{1}\left|x\left(t_{0}\right)\right|+\left|y\left(t_{0}\right)\right| \\
& \leq\left(-\alpha_{1}+1\right) M\left(t_{0}\right) \\
& <-\eta M\left(t_{0}\right)+\theta .
\end{aligned}
$$


If $M\left(t_{0}\right)=\max \left\{\left|x\left(t_{0}\right)\right|,\left|y\left(t_{0}\right)\right|,\left|z\left(t_{0}\right)\right|\right\}=\left|y\left(t_{0}\right)\right|$, then we have

$$
\begin{aligned}
\mathrm{D}^{+}(|\mathrm{y}(\mathrm{s})|)_{s=t_{0}} & \leq-\alpha_{2}|\mathrm{y}(\mathrm{t})|+|z(\mathrm{t})| \\
& \leq\left(-\alpha_{2}+1\right) M\left(\mathrm{t}_{0}\right) \\
& <-\eta M\left(\mathrm{t}_{0}\right)+\theta
\end{aligned}
$$

If $M\left(t_{0}\right)=\max \left\{\left|x\left(t_{0}\right)\right|,\left|y\left(t_{0}\right)\right|,\left|z\left(t_{0}\right)\right|\right\}=\left|z\left(t_{0}\right)\right|$, then we get

$$
\begin{aligned}
\mathrm{D}^{+}(|z(s)|)_{s=t_{0}} \leq & -\inf _{\mathrm{t} \geq 0}\left(f_{1}(\mathrm{t}, x(\mathrm{t}))-\alpha_{1}-\alpha_{2}\right)\left|z\left(\mathrm{t}_{0}\right)\right| \\
& +\sup _{\mathrm{t} \geq 0}\left|\left(f_{1}(\mathrm{t}, x(\mathrm{t}))-\alpha_{1}\right)\left(\alpha_{1}+\alpha_{2}\right)-f_{2}(t, x(t))-\alpha_{2}^{2}\right|\left|y\left(t_{0}\right)\right| \\
& +\mathrm{L}_{0}\left|x\left(t_{0}\right)\right|+\sum_{i=1}^{n} L_{i}\left|x\left(t_{0}-\tau_{i}\left(t_{0}\right)\right)\right|+\sum_{i=0}^{n} q_{i}+|p(t)| \\
\leq & \left(\sum_{i=0}^{n} L_{i}-\alpha_{3}\right) M\left(t_{0}\right)+\sum_{i=0}^{n} q_{i}+|p(t)| \\
< & -\eta M\left(t_{0}\right)+\theta .
\end{aligned}
$$

In addition, if $M\left(t_{0}\right) \geq \frac{\theta}{\eta}$, then (3.7), (3.8) and (3.9) imply that $M(t)$ is strictly decreasing in a small neighborhood $\left(t_{0}, t_{0}+\delta_{0}\right)$. This contradicts that $M(t)$ is non-decreasing. Therefore, $M\left(t_{0}\right)<\frac{\theta}{\eta}$ and

$$
\max \left\{\left|x\left(t_{0}\right)\right|,\left|y\left(t_{0}\right)\right|,\left|z\left(t_{0}\right)\right|\right\}<\frac{\theta}{\eta} .
$$

For $\forall t>t_{0}$, by the same approach used in the proof of (3.10), we have

$$
\max \{|x(t)|,|y(t)|,|z(t)|\}<\frac{\theta}{\eta}, \text { if } M(t)=\max \{|x(t)|,|y(t)|,|z(t)|\} .
$$

On the other hand, if $M(t)>\max \{|x(t)|,|y(t)|,|z(t)|\}, t>t_{0}$, we can choose $t_{0} \leq t_{3}<t$ such that $M\left(t_{3}\right)=\max \left\{\left|x\left(t_{3}\right)\right|,\left|y\left(t_{3}\right)\right|,\left|z\left(t_{3}\right)\right|\right\}<\frac{\theta}{\eta}$ and $M(s)>\max \{|x(s)|,|y(s)|,|z(s)|\}$ for all $s \in\left(t_{3}, t\right]$. Using a similar argument as in the proof of Case (I), we can show that $M(s) \equiv$ $M\left(t_{3}\right)$ is a constant, for all $s \in\left(t_{3}, t\right]$, which implies $\max \{|x(t)|,|y(t)|,|z(t)|\}<M(t)=M\left(t_{3}\right)=$ $\max \left\{\left|x\left(t_{3}\right)\right|,\left|y\left(t_{3}\right)\right|,\left|z\left(t_{3}\right)\right|\right\}<\frac{\theta}{\eta}$.

To sum up, the solutions of (1.2) are uniformly bounded. The proof is complete.

\section{An example}

Consider the following equation 


$$
\begin{aligned}
x^{\prime \prime \prime}(\mathrm{t})+(11 & \left.-\frac{1}{1+\mathrm{t}+\mathrm{x}^{2}(\mathrm{t})}\right) x^{\prime \prime}(\mathrm{t})+\left(31-\frac{4}{1+\mathrm{t}+\mathrm{x}^{2}(\mathrm{t})}\right) x^{\prime}(\mathrm{t})+\left(26-\frac{1}{1+\mathrm{t}+\mathrm{x}^{2}(\mathrm{t})}\right) x(\mathrm{t}) \\
& +\frac{1}{1+\mathrm{t}+\mathrm{x}^{2}(\mathrm{t})} x(\mathrm{t}-|\sin \mathrm{t}|)+(\sin \mathrm{t}) \sin x\left(\mathrm{t}-\mathrm{e}^{|\sin \mathrm{t}|}\right)=\frac{1}{1+\mathrm{t}^{2}} .
\end{aligned}
$$

Setting $y(t)=\frac{d x(t)}{d t}+2 x(t)$ and $z(t)=\frac{d y(t)}{d t}+2 y(t)$, we can transform (4.1) into

$$
\begin{aligned}
\frac{d x(t)}{d t}= & -2 x(t)+y(t), \\
\frac{d y(t)}{d t}= & -2 y(t)+z(t), \\
\frac{d z(t)}{d t}= & \left.-\left(7-\frac{1}{1+t+x^{2}(t)}\right) z(t)+y(t)+\frac{1}{1+t+x^{2}(t)} x(t)\right) \\
& -\frac{1}{1+t+x^{2}(t)} x(t-|\sin t|)-(\sin t) \sin x\left(t-e^{|\sin t|}\right)+\frac{1}{1+t^{2}} .
\end{aligned}
$$

Then, we can satisfy the following assumptions:

i) $\left|\left(\left(\alpha_{1}-f_{1}(t, u)\right) \alpha_{1}^{2}+f_{2}(t, u) \alpha_{1}\right) u-g_{0}(t, u)\right|=\left|\frac{1}{1+t+u^{2}} u\right| \leq L_{0}|u|+q_{0}$

ii) $\left|\frac{1}{1+t+u^{2}} u\right| \leq L_{1}|u|+q_{1}$ and $|\sin t \sin u| \leq L_{2}|u|+q_{2}$,

iii) $\alpha_{3}=\inf _{t \geq 0}\left(7-\frac{1}{1+t+u^{2}}\right)-1>\sum_{i=0}^{2} L_{i}$ by taking suitable $L_{i}$ and $q_{i}$ such as $L_{0}=L_{1}=L_{2}=1$ for appropriate $\mathrm{q}_{i}(i=0,1,2)$. Hence, all solutions of the system (4.2) are uniformly bounded.

Received: April 2011. Revised: February 2012.

\section{References}

[1] Afuwape, A.U., Omari, P., and Zanolin, F. Nonlinear perturbations of differential operators with nontrivial kernel and applications to third-order periodic boundary problems. J. Math. Anal. Appl. 143, 35-56 (1989).

[2] Andres, J. Periodic boundary value problem for certain nonlinear differential equation of the third order. Math. Slovaca 35, 305-309 (1985).

[3] Fridedrichs, K. O. On nonlinear vibrations of the third-order, in: Studies in Nonlinear Vibrations Theory, Inst. Math. Mech., New York University (1949).

[4] Rauch, L.L. Oscillations of a third order nonlinear autonomous system, in: Contributions to the Theory of Nonlinear Oscillations. Ann. Math. Stud. 20, 39-88 (1950). 
[5] Cronin, J. Some mathematics of biological oscillations. SIAM Rev. 19, 100-137 (1977).

[6] Gopalsamy, K. Stability and Oscillations in Delay Differential Equations of Population Dynamic, Mathematics and Its Applications, vol. 74, Kluwer Academic, Dordrecht (1992).

[7] Li, B. Uniqueness and stability of a limit cycle for a third-order dynamical system arising in neuron modeling. Nonlinear Anal. 5,13-19 (1981).

[8] Murakami, S. Asymptotic behavior of solutions of some differential equations. J. Math. Anal. Appl. 109, 534-545 (1985).

[9] Ademola, T. A., Ogundiran, M. O., Arawomo, P. O., and Adesina, O. A. Boundedness results for a certain third order nonlinear differential equation. Appl. Math. Comput. 216(10), 30443049 (2010).

[10] Tunç, C. and Ergören, H. On boundedness of a certain non-linear differential equation of third order. J. Comput. Anal. Appl. 12(3), 687-94 (2010).

[11] Tunç, C. Boundedness of solutions to third-order nonlinear differential equations with bounded delay. J. Franklin Inst. 347(2), 415-425 (2010).

[12] Tunç, C. On the stability and boundedness of solutions of nonlinear vector differential equations of third order. Nonlinear Anal. 70(6), 2232-2236 (2009).

[13] Tunç, C. On the boundedness of solutions of third-order delay differential equations. Differ. Equ. (Differ. Uravn.) 44(4), 464-472 (2008).

[14] Afuwape, A.U. and Castellanos, J. E. Asymptotic and exponential stability of certain thirdorder non-linear delayed differential equations: frequency domain method. Appl. Math. Comput. 216(3), 940-950 (2010).

[15] Gao, H. and Liu, B. Almost periodic solutions for a class of Liénard-type systems with multiple varying time delays. Appl. Math. Model. 34, $72-79$ (2010).

[16] Yu, Y. and Zhao, C. Boundedness of solutions for a Lienard equation with multiple deviating arguments. Elec. J. Differential Equations. 2009(14), 1-5 (2009).

[17] Burton, T.A. Stability and Periodic Solutions of Ordinary and Functional Differential Equations, Academic Press, Orland, FL (1985).

[18] Hale, J.K. Theory of Functional Differential Equations, Springer-Verlag, New York (1977).

[19] Kuang, Y. Delay Differential Equations with Applications in Population Dynamics, Academic Press, New York (1993). 